\title{
Inexact Kleinman-Newton-ADI Method with Line Search to Solve Large-Scale Algebraic CSC Riccati Equations
}

\author{
P. Benner ${ }^{1}$, M. Heinkenschloss ${ }^{2}$, J. Saak ${ }^{1}$, and $\underline{H}$. K. Weichelt ${ }^{1}$ \\ ${ }^{1}$ Max Planck Institute for Dynamics of Complex Technical Systems Magdeburg, \\ Research Group Computational Methods in Systems and Control Theory (CSC) \\ ${ }^{2}$ Rice University Houston, Texas, Department of Computational and Applied Mathematics (CAAM)
}

\section{Algebraic Riccati Equation}

$$
\mathcal{R}(X)=C^{T} C+A^{T} X+X A-X B B^{T} X=0
$$

\section{Inexact Kleinman-Newton and Line Search}

Specially tailored Newton method $\Rightarrow$ Kleinman-Newton method:

$$
\mathcal{R}^{\prime}\left(X^{(k)}\right) X^{(k+1)}=\mathcal{R}^{\prime}\left(X^{(k)}\right) X^{(k)}-\mathcal{R}\left(X^{(k)}\right) .
$$

$\Rightarrow$ Converges globally for any stabilizing $X^{(0)} \in \mathbb{R}^{n \times n}$.

\section{Problems:}

- often $\left\|\mathcal{R}\left(X^{(1)}\right)\right\| \gg\left\|\mathcal{R}\left(X^{(0)}\right)\right\|$

$\Rightarrow$ Apply line search idea [1]; BUT: involves dense computations.

- Newton step computed iteratively (necessary accuracy unknown). $\Rightarrow$ Apply inexact Kleinman-Newton idea [4] and solve

$$
\left(A^{(k)}\right)^{T} X^{(k+1)}+X^{(k+1)} A^{(k)}=-G^{(k)}\left(G^{(k)}\right)^{T}+L^{(k+1)} .
$$

BUT: How to monitor residual $L^{(k+1)}$ and check conv. conditions?

\section{Low-Rank Residual ADI [3]}

Newton step $k$ :

$$
\left(A^{(k)}\right)^{T} X+X A^{(k)}=-G^{(k)}\left(G^{(k)}\right)^{T},
$$

with $A^{(k)}=A-B B^{T} X^{(k)}$ and

$$
G^{(k)}=\left[C^{T} \mid X^{(k)} B\right] \text { until }\left\|L^{(k+1)}\right\| \leq \text { tol }_{A D /} \text {. }
$$

$\Rightarrow$ Apply low-rank residual ADI method [3].

\section{Advantages:}

- Lyapunov residual has low-rank structure:

$$
L^{(k+1)}=W W^{T}, W \in \mathbb{R}^{n \times p}, p \ll n,
$$

$\operatorname{rank}\left(L^{(k+1)}\right)=\operatorname{rank}(W) \leq \operatorname{rank}(G) \ll n$.

- Low-rank structure is key for efficiency.

\section{Algorithm:}

INPUT: $A, B, C$, and stabilizing $X^{(0)}$

OUTPUT: stabilizing spsd solution $X^{(*)}$

1. Set $k=0$ and $\bar{\eta} \in(0,1)$.

2. while $\left\|\mathcal{R}\left(X^{(k)}\right)\right\|>$ tol $_{\text {Newton }}$ do

3. $A^{(k)}=A-B B^{T} X^{(k)}, G=\left[C^{T} \mid X^{(k)} B\right]$

4. Select $\eta_{k} \leq \bar{\eta}$ and $\alpha \in(0,1-\bar{\eta})$.

5. Compute spsd $\widetilde{X}$ such that

$\left(A^{(k)}\right)^{T} \widetilde{X}+\widetilde{X} A^{(k)}=-G G^{T}+L^{(k+1)}$,

with $\left\|L^{(k+1)}\right\| \leq \eta_{k}\left\|\mathcal{R}\left(X^{(k)}\right)\right\|$.

6. Compute step size $\lambda_{k}>0$ such that $\left\|\mathcal{R}\left(\left(1-\lambda_{k}\right) X^{(k)}+\widetilde{X}\right)\right\| \leq\left(1-\lambda_{k} \alpha\right)\left\|\mathcal{R}\left(X^{(k)}\right)\right\|$.

7. Set $X^{(k+1)}=\left(1-\lambda_{k}\right) X^{(k)}+\lambda_{k} \widetilde{X}^{(k)}$

8. $k=k+1$

9. end while 10. $X^{(k)}=X^{(*)}$

\section{Contributions}

\section{Key ingredients:}

- Riccati residual as symmetric indefinite lowrank product

$$
\mathcal{R}(X)=U D U^{T} \in \mathbb{R}^{n \times n}
$$

with $U=[W \mid \Delta K] \in \mathbb{R}^{n \times(p+q)}, D=\left[\begin{array}{cc}I_{p} & 0 \\ 0 & -l_{q}\end{array}\right]$, and $p+q \ll n$.

- Line search for large-scale case.

$\Rightarrow$ Exploits low-rank structure of residuals.

$\Rightarrow$ Highly efficient computations only use "small" inner products of low-rank factors.

$\Rightarrow$ Direct monitoring of $\|\mathcal{R}(X)\|$ and $\|L\|$.

\section{Convergence results:}

- Convergence proof in [4] is not feasible in the low-rank case.

- Use inexact Kleinman-Newton-ADI with line search and the following assumptions:

- $(A, B)$ stabilizable.

- $(C, A)$ detectable.

- $\lambda_{k}$ is bounded away from 0 .

- $X^{(k)}$ spsd \& stabilizing $\forall k \geq K>0$.

$\Rightarrow X^{(k)} \rightarrow X^{(*)}$, the unique stabilizing solution.

\section{D Heat Equation Example $(n=529, p=26, q=25)$}
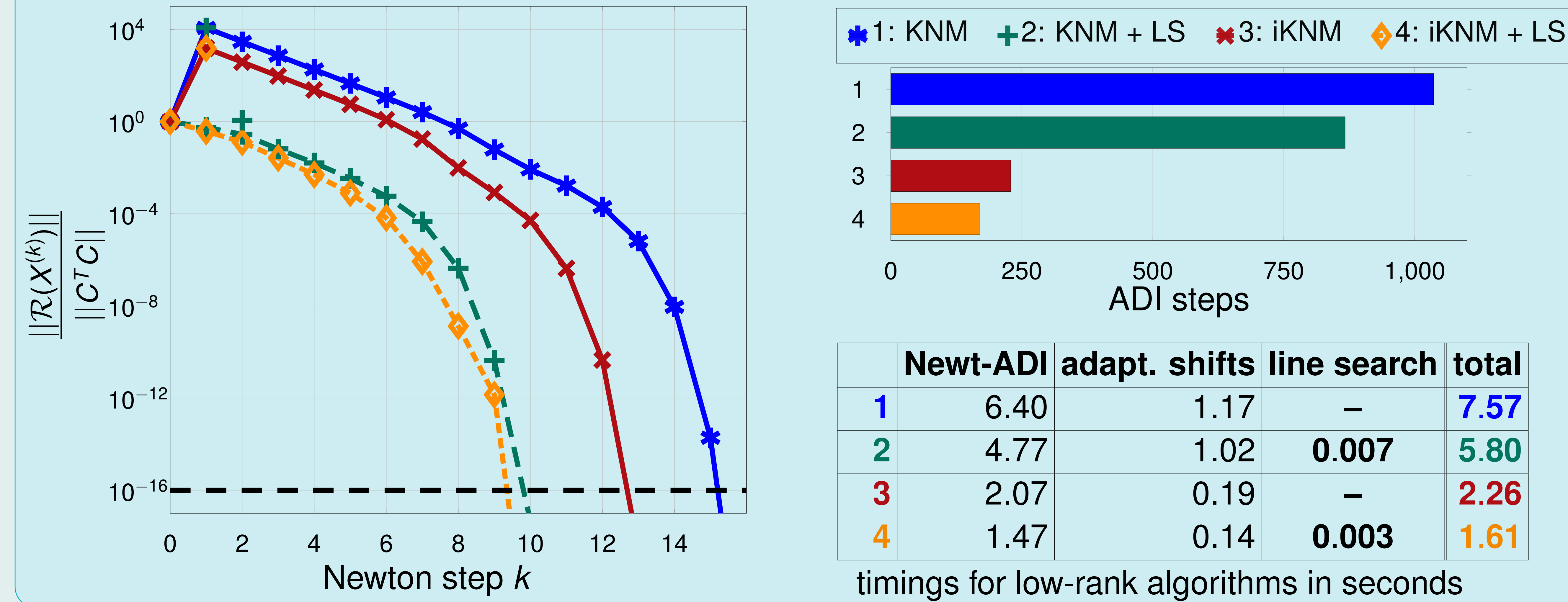

\begin{tabular}{|r|r|r|c|c|}
\hline & Newt-ADI & adapt. shifts line search & total \\
\hline $\mathbf{1}$ & 6.40 & 1.17 & - & $\mathbf{7 . 5 7}$ \\
\hline $\mathbf{2}$ & 4.77 & 1.02 & $\mathbf{0 . 0 0 7}$ & 5.80 \\
\hline $\mathbf{3}$ & 2.07 & 0.19 & - & $\mathbf{2 . 2 6}$ \\
\hline 4 & 1.47 & 0.14 & $\mathbf{0 . 0 0 3}$ & 1.61 \\
\hline timings for low-rank algorithms in seconds
\end{tabular}

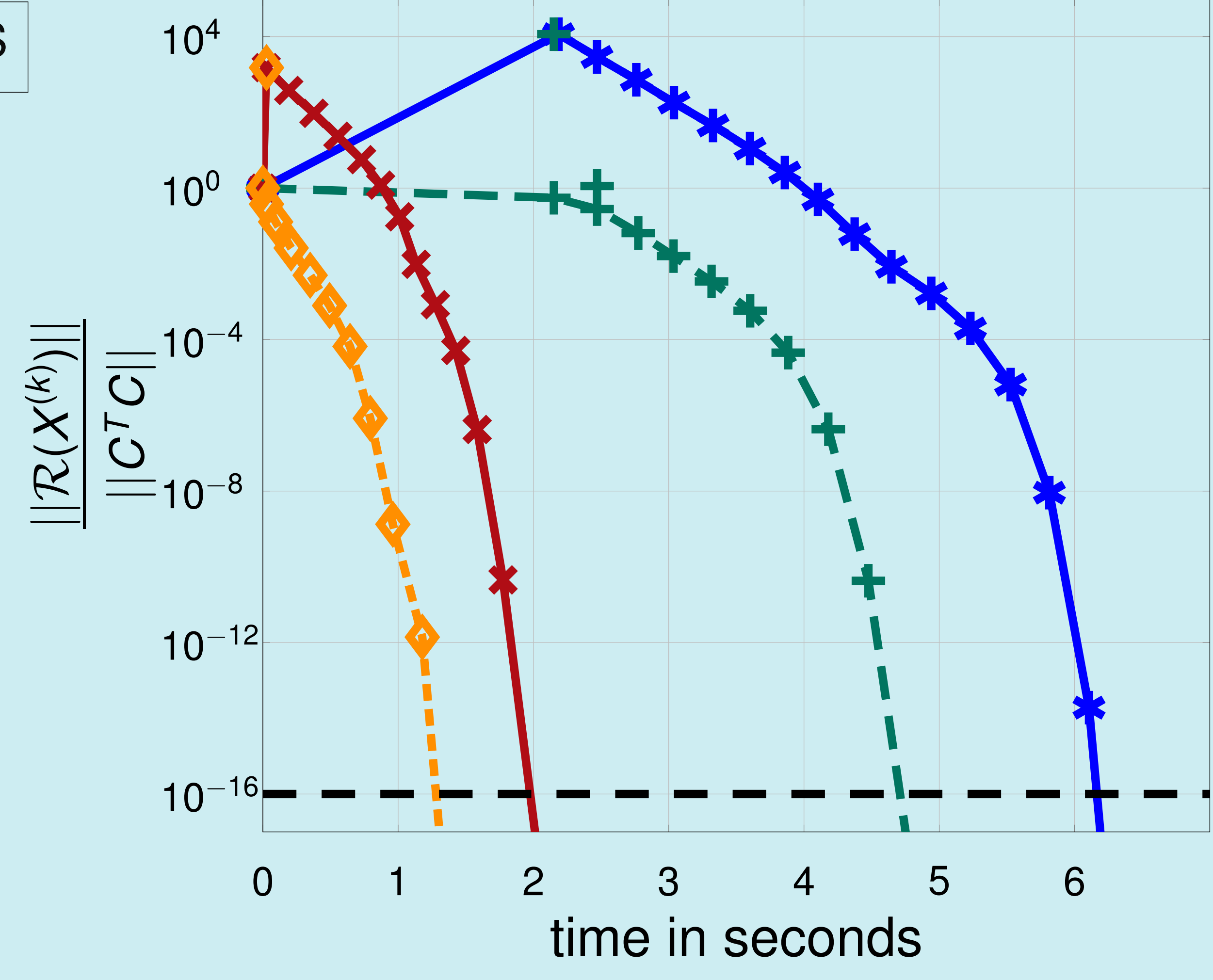

\section{References}

[1] P. BENNER AND R. BYERS, An exact line search method for solving generalized continuous-time algebraic Riccati equations, IEEE TAC, 43 (1998).

[2] P. Benner, M. Heinkenschloss, J. SaAk, AND H. K. Weichelt, Inexact low-rank Newton-ADI method for large-scale algebraic Riccati equations, Preprint MPIMD/15-06, Max Planck Institute Magdeburg, May 2015.

[3] P. BeNNER, P. KÜRSCHNER, AND J. SAAK, An improved numerical method for balanced truncation for symmetric second order systems, MCMDS, 19 (2013).

[4] F. FeItZINGeR, T. HyLLA, AND E. W. SACHS, Inexact Kleinman-Newton method for Riccati equations, SIMAX, 31 (2009). 